\title{
Toxicological assessment of additively manufactured methacrylates for medical devices in dentistry
}

\author{
Frank Alifui-Segbaya ${ }^{1^{*}}$ Jasper Bowman ${ }^{2}$ Alan R. White ${ }^{3}$ Sony Varma ${ }^{4}$ \\ Graham J. Lieschke ${ }^{5+}$ and Roy George ${ }^{6+}$ \\ DOI: https://doi.org/10.1016/j.actbio.2018.08.007
}

\footnotetext{
${ }^{1}$ School of Dentistry and Oral Health, Griffith Health, Gold Coast campus, Griffith University, QLD 4222, Australia. Email: f.alifui-segbaya@griffith.edu.au

${ }^{2}$ School of Environment and Science, Griffith Sciences, Nathan campus, Griffith University, QLD 4111, Australia. Email: jasper.bowman@griffith.edu.au

${ }^{3}$ School of Environment and Science, Griffith Sciences, Nathan campus, Griffith University, QLD 4111, Australia. Email: alan.white@griffith.edu.au

${ }^{4}$ Australian Regenerative Medicine Institute, Monash University, Clayton, Victoria 3800, Australia. Email: sony.varma@monash.edu

${ }^{5}$ Australian Regenerative Medicine Institute, Monash University, Clayton, Victoria 3800, Australia. Email: graham.lieschke@monash.edu

${ }^{6}$ School of Dentistry and Oral Health, Griffith Health, Griffith University, Australia. Email: r.george@griffith.edu.au

" Corresponding Author

Frank Alifui-Segbaya

School of Dentistry and Oral Health,

Gold Coast campus, Griffith University, Australia.

Email: f.alifui-segbaya@griffith.edu.au

† These authors contributed equally to this work
} 
Graphical Abstract

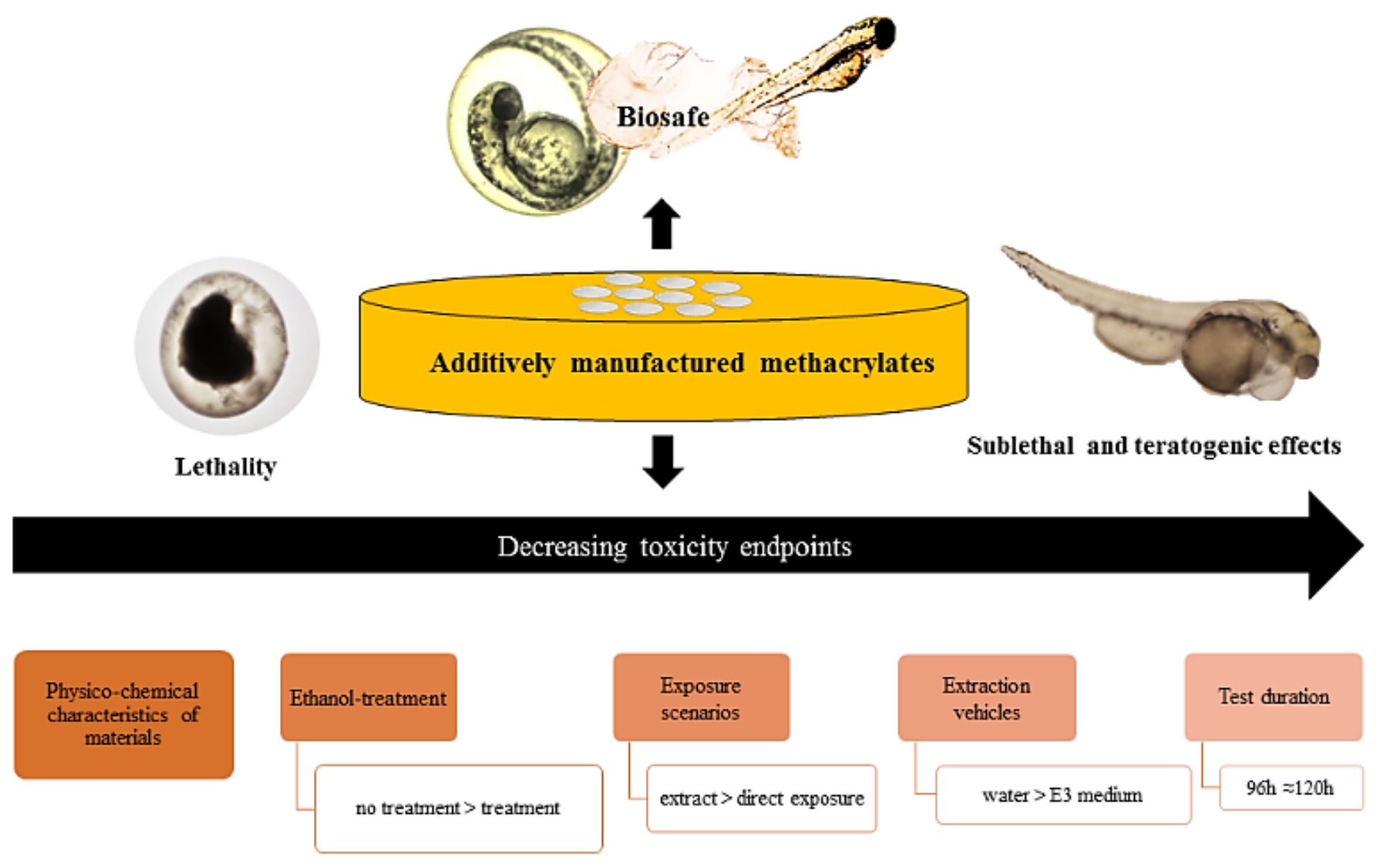




\begin{abstract}
The paucity of information on the biological risks of photopolymers in additive manufacturing is a major challenge for the uptake of the technology in the construction of medical devices in dentistry. In this paper, the biocompatibility of methacrylates for denture bases, splints, retainers, and surgical guides were evaluated using the innovative zebrafish embryo model, which is providing a high potential for toxicity profiling of photopolymers and has high genetic similarity to humans. Toxicological data obtained confirmed gradations of toxicity influenced by ethanol treatment, exposure scenarios and extraction vehicles. In direct exposure tests, juvenile fish exposed to non-treated methacrylates in ultrapure water showed accelerated toxicity endpoints compared to fish in transparent E3 medium. Similarly, toxic extracts induced mostly acute responses (embryonic mortality) in contrast to cumulative chronic (sublethal and teratogenic effects) in direct exposure. Methacrylates composed of $>60 \%$ Ethoxylated bisphenol A dimethacrylate produced a relatively lower conversion rate in FTIR spectroscopy but were safe in zebrafish bioassays after ethanol treatment. The study affirms that biocompatibility was influenced primarily by physicochemical characteristics of the materials, which subsequently influenced their residual monomer content before and after immersion in ethanol. Given the precautionary implications of the study, we propose a 3-tiered approach i.e. using approved materials, apposite manufacturing parameters and post-processing techniques that together guarantee optimal results for medical devices.
\end{abstract}

\title{
Keywords
}

Biocompatibility; Methacrylates; Zebrafish embryo model; FTIR spectroscopy; Double bond conversion; Additive manufacturing 


\section{Statement of significance}

This study is timely and relevant since there is limited published literature that precisely describes the toxicological properties of additively manufactured methacrylates despite their increased popularity for medical devices. While it is generally accepted that the zebrafish excels as a model system for developmental toxicity, a further examination of its utility in this study using different protocols provides basis for its consideration and adoption at a crucial time when there is a lack of consensus regarding the most suited biological assessment methods for medical devices. 


\section{Introduction}

Whereas natural polymers have existed since the dawn of time, it was the revolutionary discovery of synthetic polymers in the 19th century that has led to plastics becoming an essential element of modern life $[1,2]$. Current advancements in additive manufacturing (AM) or threedimensional printing (3DP) is also amplifying interest in the use of versatile synthetic polymers for bespoke medical devices. However, in dentistry, the paucity of information on the biological risks of the materials is a major challenge for the uptake of 3DP technologies. This is because photopolymerization in $3 \mathrm{DP}$ is an iterative process that may result in undesired material chemistries for some polymer systems. The structural integrity of the devices may likewise vary when they are built with different printers or even when the same printer, parameters, process steps, and materials are used [3]. For acrylic devices, there is a propensity for them to accumulate residual monomer and degradation products that can cause local and systemic side effects in high doses [4-6]. To put this in context, intraoral devices can cause cytotoxic effects on pulp and oral mucosa, and inhibit protein synthesis of the oral epithelial cells [7]. Also, apart from the medical applications of acrylic esters, their widespread use in the production of plastics for food packaging, surface coatings and emulsion polymers also raises questions pertinent to their toxicological properties, and their short-and long-term health effects (e.g. allergenic reactions or chemical "burns") on persons exposed to them $[8,9]$. As such, the adverse effects of simple and multifunctional esters have been the focus of many toxicological studies [8-12]. In this paper, the biocompatibility of additively manufactured methacrylates are evaluated using the innovative zebrafish (Danio rerio) embryo model [13, 14], which is providing a high potential for toxicity profiling of photopolymers [15-22] and has high genetic similarity to humans [23]. In general, the zebrafish is an excellent model for developmental toxicity and offers advantages such as external fertilization, high fecundity, transparent embryos that permit 'whole organism' assessment through direct observation of internal organs and ease of phenotype assessment over other vertebrates or rodents models, in which aspects of organogenesis and disease pathology cannot be examined without interventions such as surgery or postmortem examination [24]. To our knowledge, there is no reported study of long-term photopolymers using the zebrafish as a model organism to test toxicity. 


\section{Materials and Methods}

Representative materials for experimentation were built with EnvisionTec's digital light processing (DLP) [25] and Formlab's reverse stereolithography (SL) [26] systems. Samples comprised E-Denture (ED), E-Guard (EG) and Dental SG (DSG) methacrylates. ED is a Class IIa acrylic material for 3DP of denture bases containing $>60 \%$ Ethoxylated bis-phenol A dimethacrylate (Bis-EMA), 15-25\% Proprietary methacrylic oligomer and $<2,5 \%$ Phenyl bis(2,4,6-trimethylbenzoyl)-phosphine oxide, as w/w\% hazardous ingredients [27]. At the time of commencing this study, ED did not receive FDA approval for its intended purpose. EG is a Class I material ( $>70 \%$ Proprietary methacrylic oligomer, $<20 \%$ glycol methacrylate, $<5 \%$ Pentamethyl-piperidyl sebucate and $<2,5 \%$ Diphenyl(2,4,6-trimethylbenzoyl)phosphine oxide))[28] for 3DP of splints and retainers. DSG is a Class I material ( $\geq 75 \%$ Bis-EMA, 30-

$50 \%$ Diurethane dimethacrylate, mixture of isomers and $<10 \%$ phenyl bis $(2,4,6-$ trimethylbenzoyl)-phosphine oxide) [29] indicated for 3DP of surgical guides and diagnostic models.

\subsection{Sample preparation}

Disk-shaped 60 x 3 mm ED and EG samples were built by EnvisionTec GmbH (Brüsseler Str. 51, 45968 Gladbeck, Germany) with their recommended Perfactory ${ }^{\circledR}$ DDP 4M 3D printer (Zheight: $67.98 \mathrm{~mm}$; Voxel: $100 \mathrm{~lm}$; Light power: $180 \mathrm{Mw} / \mathrm{dm} 2)$ and postcured (2 x 100 flashes) in Otoflash G171 (NK-Optik GmbH, Isarstr. 2, D-82065 Baierbrunn, Germany). The samples were tested in ethanol-treated $(\mathrm{Tx})$ and non-treated $(\mathrm{nTx})$ conditions. Tx samples were soaked in ethanol absolute (Purity $\geq 99.9 \%$, Merck KGaA, Darmstadt, Germany) for 3 min, rinsed five times with ISO 3696 grade 1 water and air-dried for $2 \mathrm{~h}$ at ambient temperature. DSG samples, on the other hand, were built in-house from Dental SG (DSG) V1 resin (Formlabs Inc. 35 Medford St. Suite 201, Somerville, MA 02143, USA) with the recommended Form 2 reverse SL 3D Printer (laser specifications: $405 \mathrm{~nm}$ violet laser and 250mW laser; Laser Spot Size: $140 \mathrm{~mm}$; Layer thickness, Dz: $50 \mathrm{~mm}$ ). Samples comprised Tx, nTx and as-built (aSb) or 'green' (FIGURE 1) disks that were not postcured after they were rinsed in isopropanol (Purity 99.5\%, Acros 
Organics ENA23, zone1, Janssen Pharmaceuticalaan 3a, B-2440 Geel, Belgium) to remove resin remnants. Postcuring of $\mathrm{n}(\mathrm{Tx})$ samples was completed in LC-3DPrint Box (Vertex-Dental, B.V. Centurionbaan 190, 3769 AV Soesterberg, The Netherlands) as recommended by the manufacturer [30].

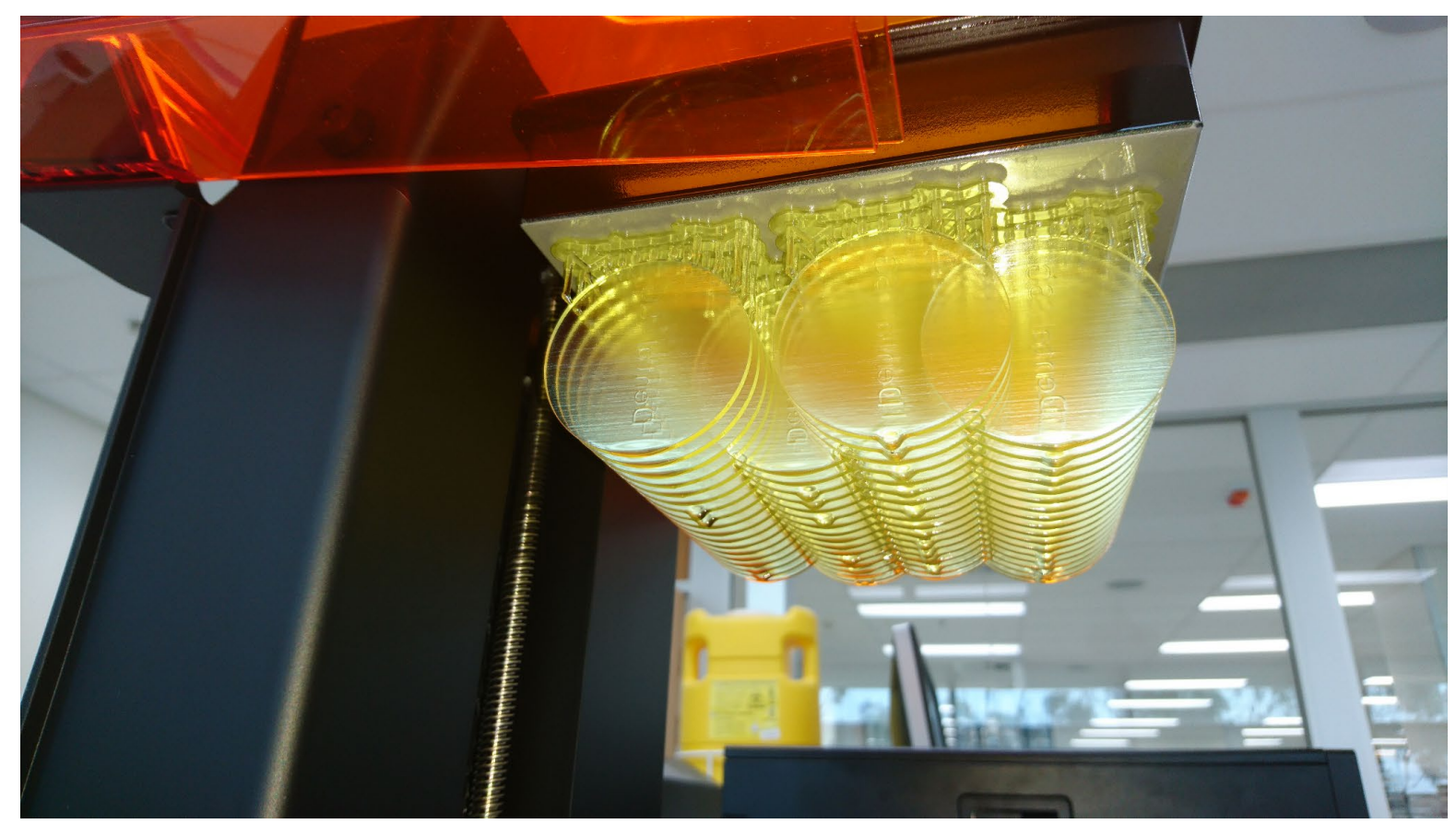

FIGURE 1: aSb (green) disks built from DSG liquid photopolymer resin in Form 2 SL 3D printer.

\subsection{Experimentation with zebrafish embryo model}

Embryos for experiments were obtained from 5 to 6 months old wild-type zebrafish (AB/Tü strain) by pair-wise mating and natural spawning. The fish culture (FishCore, Australian Regenerative Medicine Institute, Monash University) was maintained at $28{ }^{\circ} \mathrm{C}, \mathrm{pH}$ of $7.2,14 \mathrm{~h}$ light/10 h dark photoperiod and fed with Artemia and NRD marine diet. Ethical approval (MARP/2015/094) to use zebrafish for experiments was issued by the Animal Ethics Committee in Monash University, Australia.

In the preliminary biological test, randomly selected 16-cell stage or 1.5-h postfertilized (hpf) embryos $(n=10)$ were cultured on ED and EG samples in soda lime petri dishes with lid 
(Brand ${ }^{\circledR} \mathrm{GmbH}+\mathrm{Co}$ Kg, Otto-Schott-Straße 25, 97877 Wertheim, Germany) using transparent E3 medium $(\mathrm{E} 3 \mathrm{M})[15,18]$ and ultrapure water $(\mathrm{UW})[17,19]$ as extraction vehicles. The ratio of surface-area-of-material to volume-of-extraction vehicle used is in accordance with the standards [31]. Two test batches including controls were incubated in Heracell $\mathrm{CO}_{2}$ incubator (Thermo Fisher Scientific Inc.) at an optimum temperature of $28.5^{\circ} \mathrm{C}$. Fish in the first batch were assessed at $24 \mathrm{~h}$ intervals for lethal endpoints (embryo coagulation, absence of somite formation, non-detachment of tail-bud and absence of heartbeat) until $96 \mathrm{~h}$ [13] using Olympus MVX10 Research Macro Zoom Microscope, Olympus DP 72 digital colour microscope camera and cellSens imaging software (Olympus Soft Imaging Solutions GmbH) (MMI, Monash University, Australia). Fish in bioassays were also assessed for sublethal developmental endpoints (development of eyes, spontaneous movement, hypopigmentation and formation of edemata) and endpoints of teratogenicity (spinal curvature, malformed tail and yolk deformation)[32]. Fish in the second batch were incubated continuously for $120 \mathrm{~h}$ before a oneoff phenotype assessment including growth retardation. For each methacrylate sample, 40 embryos were incubated and assessed including repeat tests. In the additional biological test, embryos were cultured in extracts $\left(37 \pm 1^{\circ} \mathrm{C}\right.$ in UW for $24 \mathrm{~h}$ at $3 \mathrm{~cm}^{2}: 1 \mathrm{~mL}$ ratio [33]) obtained from nTx, Tx and aSb DSG samples. Toxicological data from the extract tests were compared with data from direct exposure tests with the same samples, before and after leachate extraction. The bioassays in this group were assessed at $24 \mathrm{~h}$ intervals for developmental endpoints. At the end of the test, surviving fish were euthanized in $0.4 \%$ anaesthetic tricaine mesylate solution. 


\section{Results}

By day 3, a 100\% hatching rate was recorded in controls and bioassays containing $\mathrm{Tx}$ methacrylates. The results in this study are valid as the survival rate in controls was $\geq 90 \%$ with no developmental endpoints[13]. For clarity, "embryo" denotes 24 - 72 hpf juvenile fish, "larva" denotes 96 - 120 hpf juvenile fish and without regard to exceptions, both are referred to as "fish". TABLES 1 AND 2 show cumulative endpoints observed in bioassays when tests were concluded. Supplementary files (S1-S16) are selected video recordings of fish in bioassays.

\subsection{Toxicological report for ED methacrylate}

On day 1, nTx ED in E3M and UW induced 10\% and 20\% lethality in bioassays, respectively, in addition to hypopigmentation and behavioural perturbations. On day 2, embryos exposed to nTx ED in E3M developed severe yolk sac and/or pericardial edema with a few tail malformations whereas embryos in UW recorded additional 60\% lethality with severe malformations and test was concluded. On day 3, embryos in E3M were markedly hypopigmented (albino), lethargic with yolk sac edema and deformations, and pericardial edema. For the duration of the test, no lethal endpoint was observed in bioassays containing Tx ED; however, larvae in one of the UW assays developed minor pericardial edema, dark yolk sacs and reduced melanophores. At 120 h, nTx ED (including nTx EG) assays recorded a 100\% lethality (embryonic mortality). Tx ED assays recorded a $100 \%$ survival rate with no developmental endpoint. Larvae in E3M (S1) were phenotypically comparable to control larvae (S2); however, 30\% of larvae in UW (FIGURE 2 AND S3) developed minor pericardial edema. 


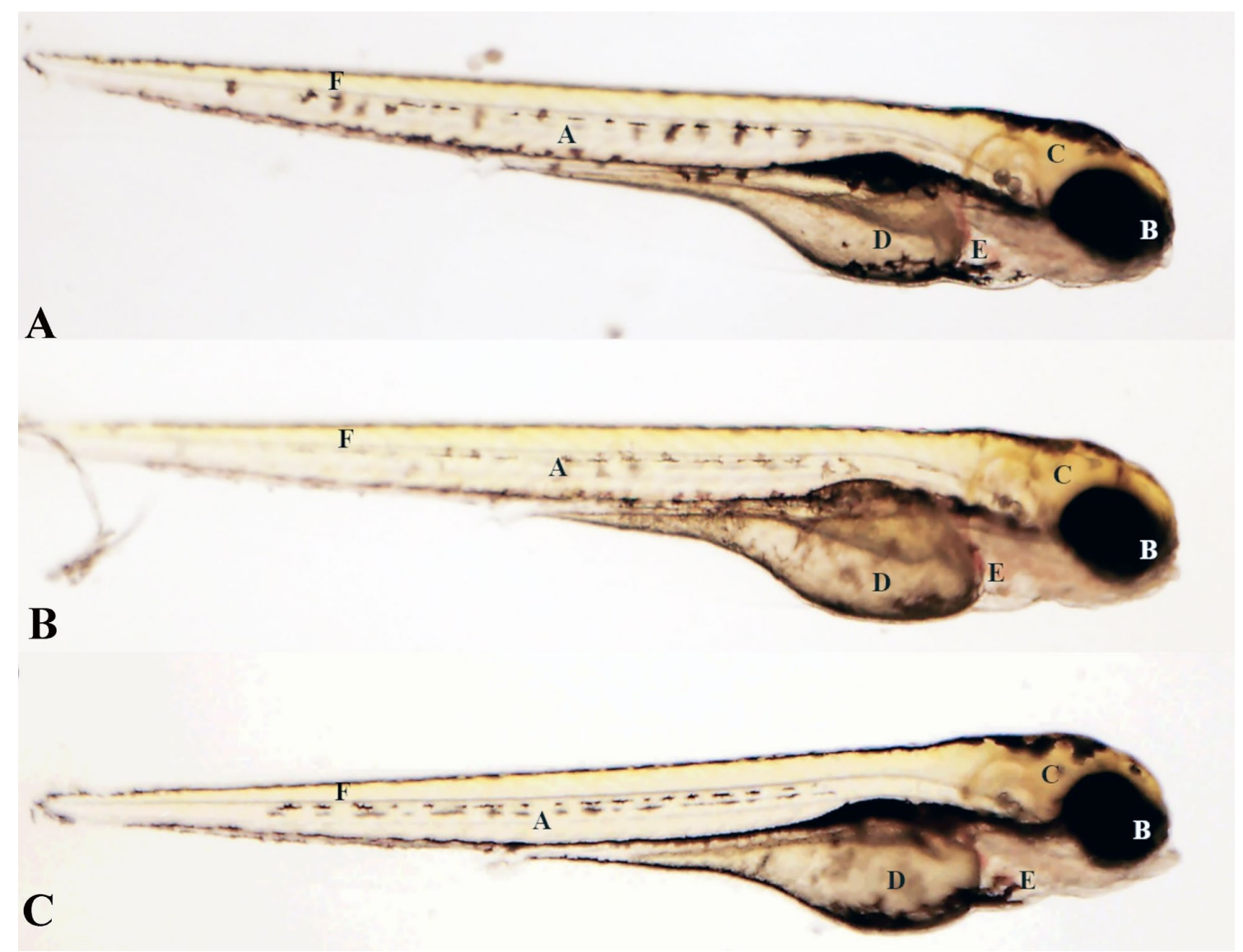

FIGURE 2: Day 5 representative larvae exposed to E-Denture (A) and E-Guard (B) in water in comparison to Control (C). Note the phenotype similarities and differences in larvae: Body (A) Eye (B) Head (C) Yolk sac (D) Pericardial sac (E) and Tail (F).

\subsection{Toxicological report for EG methacrylate}

By day 2, nTx EG in E3M induced > 50\% lethality in bioassays, in addition to hypopigmentation, behaviour perturbations and yolk deformation (S4). For nTx EG in UW, $>50 \%$ lethality with severe hypopigmentation, behaviour perturbations and malformations was observed on day 1 and tests were discontinued. For bioassays containing Tx EG, one coagulated embryo (E3M) and tail malformation (UW) were observed on days 1 and 3, respectively.

On day $4, \approx 50 \%$ of the larvae in both E3M and UW assays developed minor pericardial edema, dark yolk sacs and reduced melanophores. At 120 h, bioassays containing Tx EG recorded a 90\% survival rate with no indicators of lethality but larvae in both E3M (S5) and UW (S6) showed reduced melanophores and minor pericardial edema. 


\subsection{Toxicological report for DSG material}

Extracts from aSb and nTx (S7) DSG samples elicited a 100\% lethality (embryonic mortality) in bioassays within $24 \mathrm{~h}$. In comparison to direct exposure, aSb DSG also induced a 100\% lethality (embryonic lethality) within $24 \mathrm{~h}$. Embryos exposed to nTx DSG survived but showed cumulative lethargy, pericardial edema and yolk deformation after $48 \mathrm{~h}$, and test was discontinued. Likewise, surviving embryos exposed to nTx DSG after extraction (FIGURE 3), were sternly lethargic with severe pericardial edema and yolk sac edema by $72 \mathrm{~h}$. No developmental endpoint was observed in Tx and control assays. S8-S12 show embryonic development in some of the bioassays. FIGURE 4 shows larvae length in UW and E3M bioassays after $120 \mathrm{~h}$ continuous exposure to Tx ED and Tx EG in comparison to controls. 


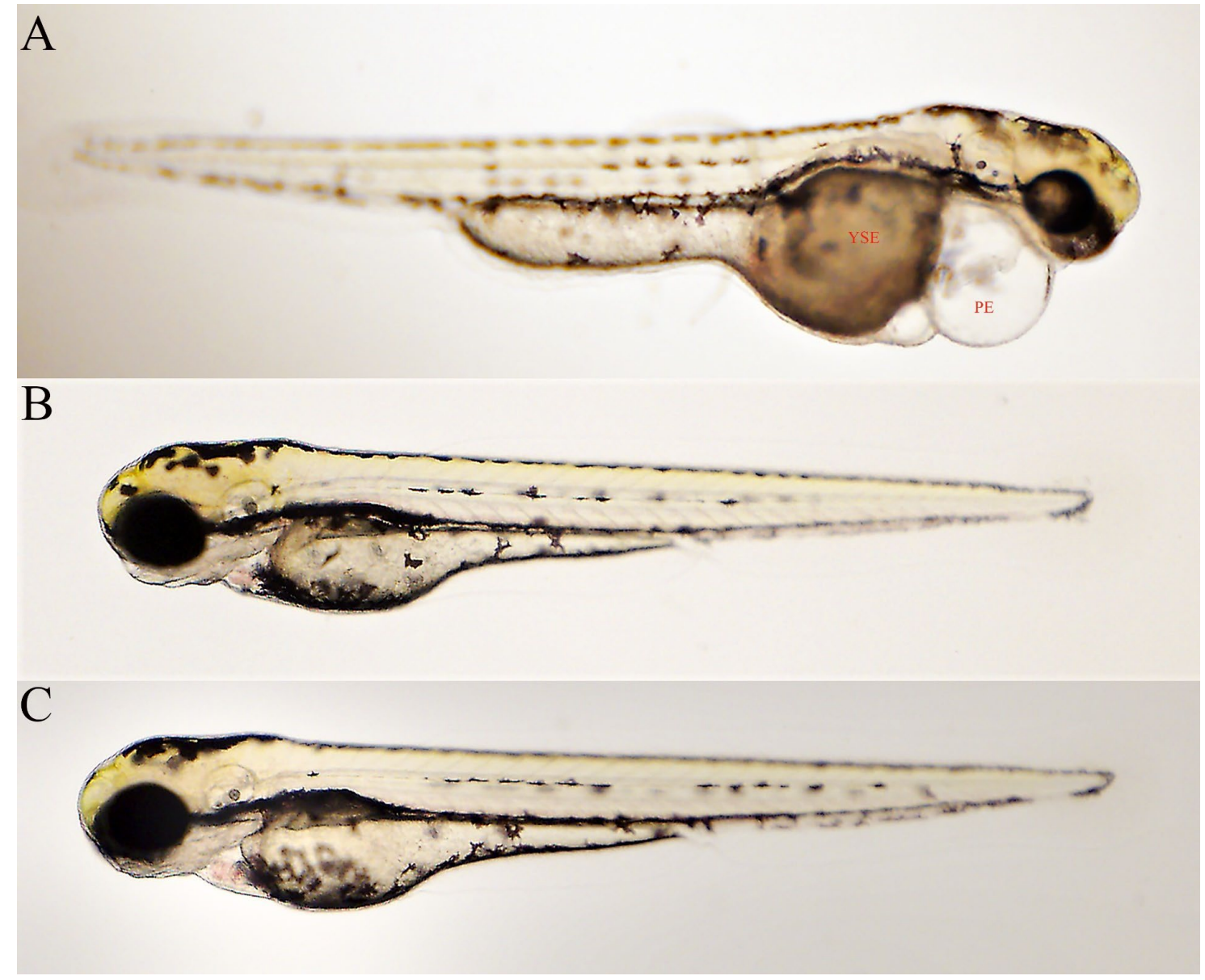

FIGURE 3: Day 5 representative larvae exposed to E-Denture (A) and E-Guard (B) in water in comparison to Control (C). Note the phenotype similarities and differences in larvae: Body (A) Eye (B) Head (C) Yolk sac (D) Pericardial sac (E) and Tail (F). 


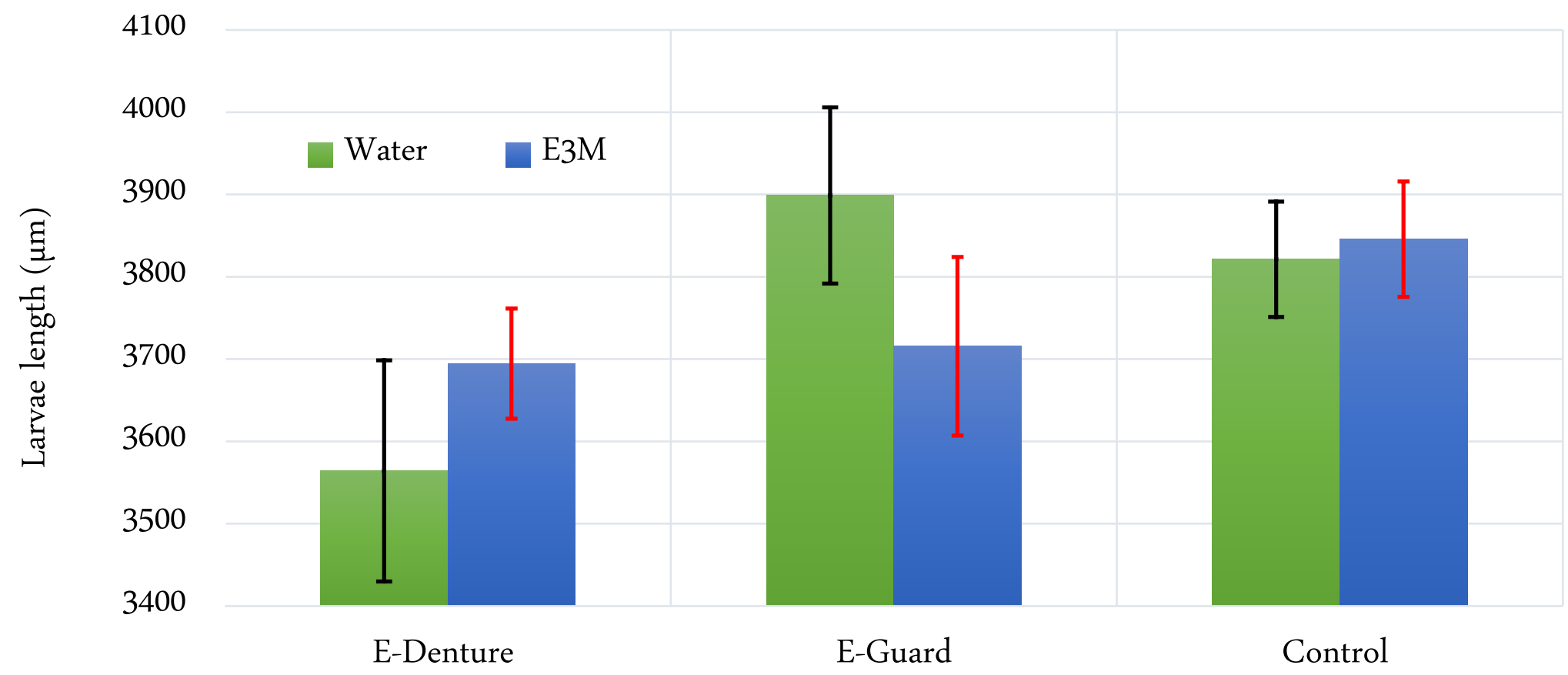

FIGURE 4: Larvae length of surviving fish exposed to E-Denture and E-Guard in comparison to fish in Controls after 120 h continuous exposure in water and E3 medium. Error bars show standard deviation from the mean. 
TABLE 1 Cumulative toxicity endpoints observed in E-Denture (ED) and E-Guard (EG) bioassays.

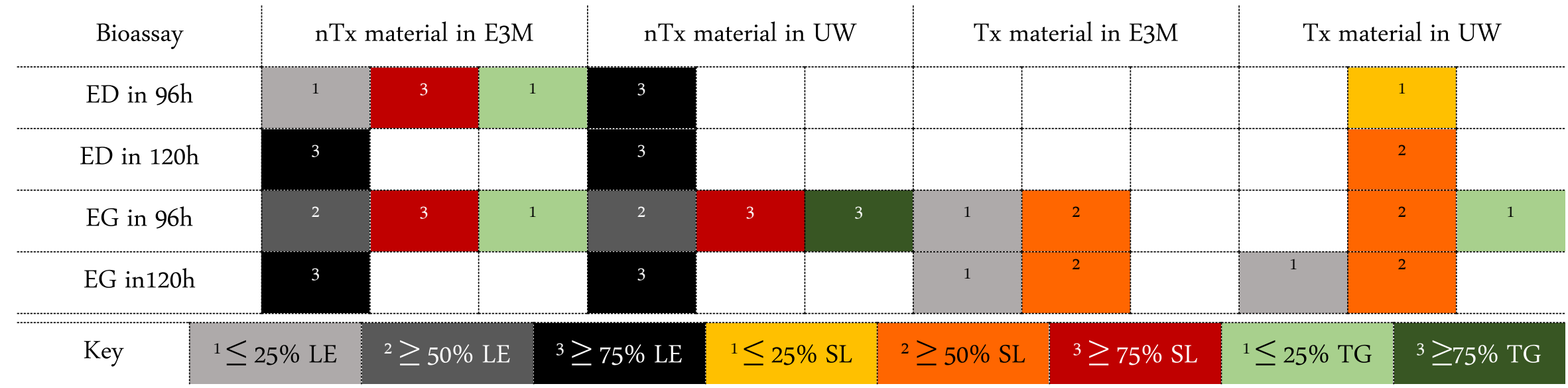

TABLE 2 Cumulative toxicity endpoints observed in Dental SG (DSG) bioassays.

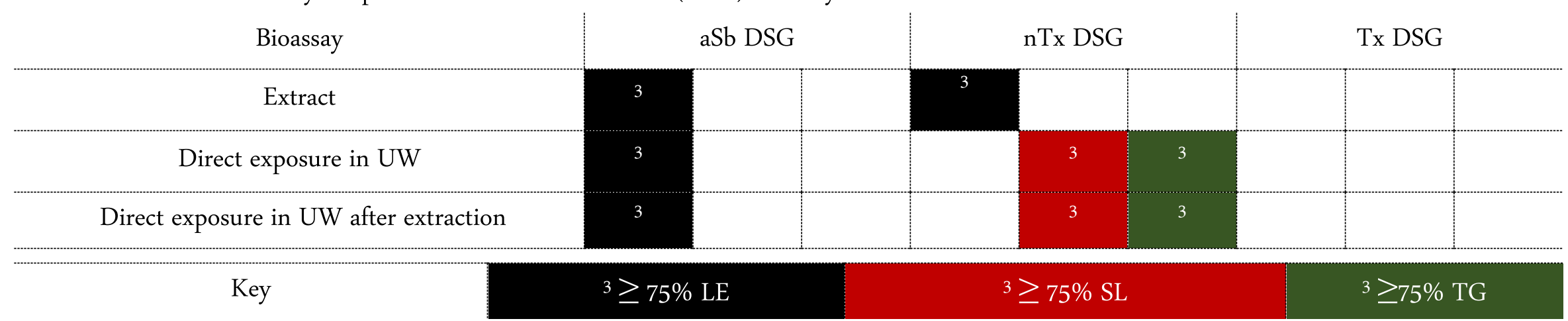

aSb As-built nTx Non-treated Tx Ethanol-treated E3M E3 medium UW Ultrapure water

LE Lethal endpoints SL Sublethal developmental endpoints TG Endpoints of teratogenicity 


\subsection{Characterization of double bond conversion}

Preliminary degree of conversion (DC I) was quantified to provide information on unreacted carbon-carbon $(\mathrm{C}=\mathrm{C})$ double bond in photocured methacrylates (FIGURE 5). DC (\%) was calculated based on the ratio of variable absorbance of aliphatic double bond (peaks at 1637 $\mathrm{cm}^{-1}$ ) against internal standard, which is constant absorbance of aromatic double bond (peaks at $1608 \mathrm{~cm}^{-1}$ ) (peaks at $1608 \mathrm{~cm}^{-1}$ ) [7] as shown in the formula below:

$$
\mathrm{DC}(\%)=1-\frac{\text { Abs in photocured resin }(\text { aliphatic } \mathrm{C}=\mathrm{C}) /(\text { aromatic } \mathrm{C}=\mathrm{C})}{\text { Abs in liquid resin }(\text { aliphatic } \mathrm{C}=\mathrm{C}) /(\text { aromatic } \mathrm{C}=\mathrm{C})} \times 100
$$

DC in representative materials $(\mathrm{n}=6)$ was measured using Perkin Elmer Spectrum Two FT-IR with Universal ATR for contact with the diamond/ZnSe crystal (PerkinElmer, Inc. 940 Winter Street Waltham, MA 02451 USA) at $4 \mathrm{~cm}^{-1}$ spectral resolution, 4 scans and $4000-450 \mathrm{~cm}^{-1}$ range. We maintained absorbance at $1608 \mathrm{~cm}^{-1}$ peaks for EG despite minor fluctuations since attempts to resolve at $1530 \mathrm{~cm}^{-1}$ peaks produced inconsistent results. For ED and EG, comparable NextDent Denture [34] and Dental LT Clear [35], liquid photopolymer resins were used, respectively. Alternative DC (DC II) was calculated following polymerization reactions in liquid photopolymer resins.

During experimentation, resin drops were placed in transparent polyethylene films and pressed between sodium chloride crystals to form $0.02 \mathrm{~mm}$ thick samples upon irradiation at $20 \mathrm{~mm}$ fixed distance using Radii-cal LED light (SDI Limited, Bayswater, Victoria 3153, Australia). Sample irradiation was within $440-480 \mathrm{~nm}$ wavelength range and $1200 \mathrm{~mW} / \mathrm{cm}^{2}$ light intensity. The degree of conversion ( $\mathrm{x}$ ) was calculated from the decrease in IR absorbance (A) after a given exposure using the formula: $x=\left(A_{0}-A_{t} / A_{0}\right) \times 100 ; A_{0}$ is peak absorbance at $1637 \mathrm{~cm}^{-1}$ at null time (initial intensity when time is equal to zero), and $A_{t}$, which is the absorbance at time, $\mathrm{t}$ (measured intensity over time)[36, 37]. Experiments were performed in triplicates. Mean values obtained at different time periods $(20,40,60,80,100,120,140,160,180$ and $200 \mathrm{~s})$ are shown in FIGURE 6. 


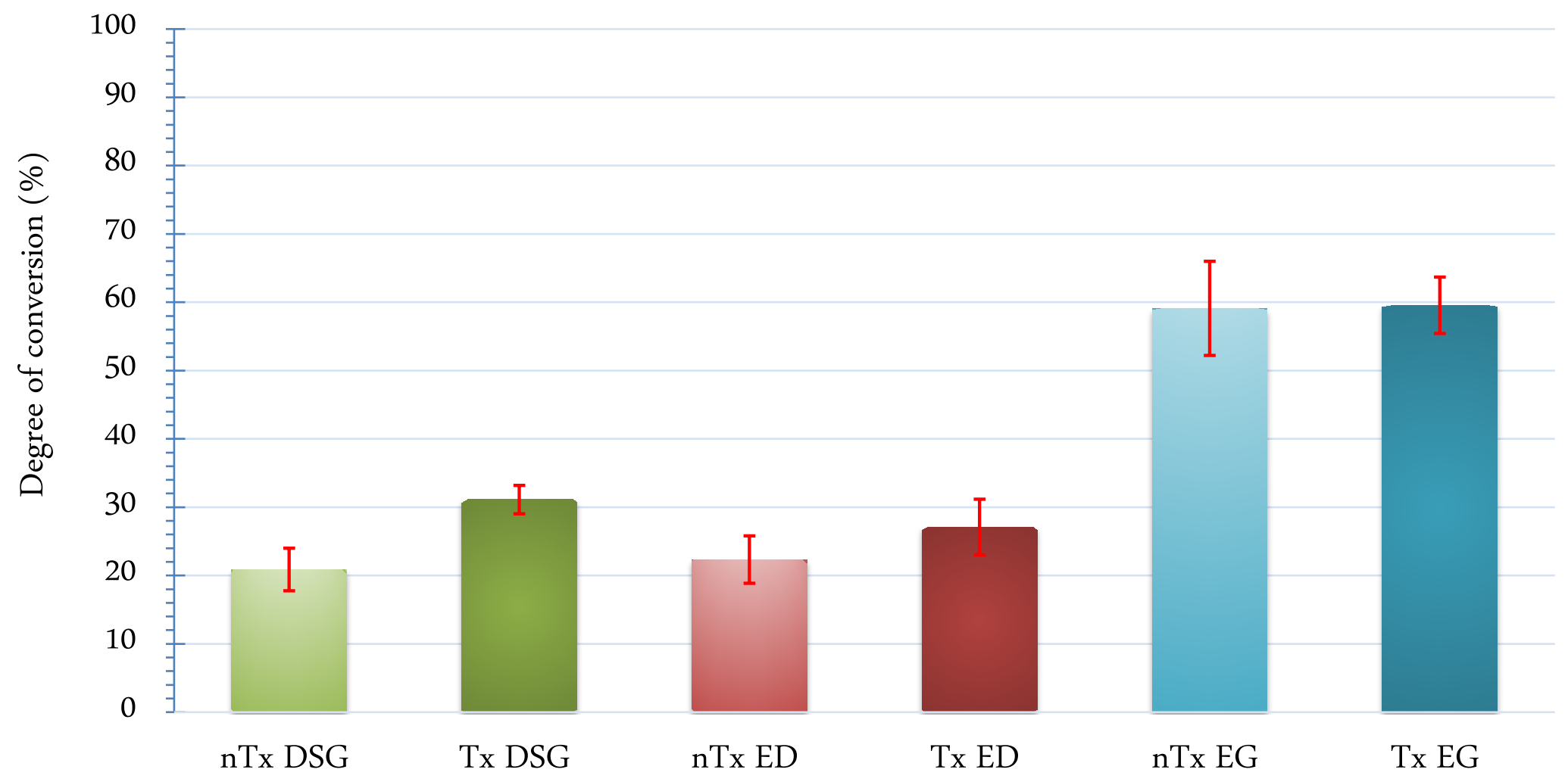

FIGURE 5: Conversion rates in non-treated (nTx) versus ethanol-treated (Tx) Dental SG (DSG), E-Denture (ED) and E-Guard (EG) methacrylates. Error bars show standard deviation from the mean. 




FIGURE 6: Time-dependent conversion rate measured in Dental SG (DSG), E-Denture (ED) and E-Guard (EG) liquid photopolymer resins. Error bars show standard deviation from the mean. 


\subsection{Statistical analysis}

A two-way ANOVA test for larvae length showed there was a main effect of material and significant interaction between material and extraction vehicle; t-test showed significant difference ( $p \leq 0.05$, unpaired, two-tailed) within the sample groups. For preliminary degree of conversion (DC I), significant difference ( $\mathrm{t}$-test, $p \leq 0.05$, unpaired, two-tailed) exists within treatment groups for DSG and ED materials. 


\section{Discussion}

\subsection{Toxicological endpoints}

Behaviour perturbation, mostly lethargy, was a common sublethal endpoint observed in our toxicity assays, consistent with a previous report examining polymer extracts in this assay [19]. Russom et al. [11] reported hypoactive locomotor activity in juvenile fathead minnows exposed to methacrylates. Mammals are also sensitive to behavioural effects of these chemicals: doserelated increase in sleeping time was reported in mice exposed to methyl methacrylate and ethyl methacrylate vapours [38]. Although relatively non-specific, some of the developmental endpoints we observed in toxic bioassays (FIGURE 7, S13 AND S14) are comparable to those reported in animal studies that linked methacrylic esters to embryonic fetal toxicity, teratogenicity [8] and cardiovascular function $[4,6]$. In direct exposure tests, embryos cultured on materials with water (S15) showed accelerated toxicological effects compared to fish in E3M (S16). The delayed toxicological effect in E3M bioassays may be due to additional supply of calcium to dechorionated embryos [39]. Toxic extracts induced mostly acute responses (embryonic mortality) in contrast to cumulative chronic (sublethal and teratogenic endpoints) in direct exposure. Although $120 \mathrm{~h}$ fish culture produced comparable toxicological data as 96 h culture, daily assessment in the latter ensures dead or compromised embryos removed since they can decompose rapidly [40]. 


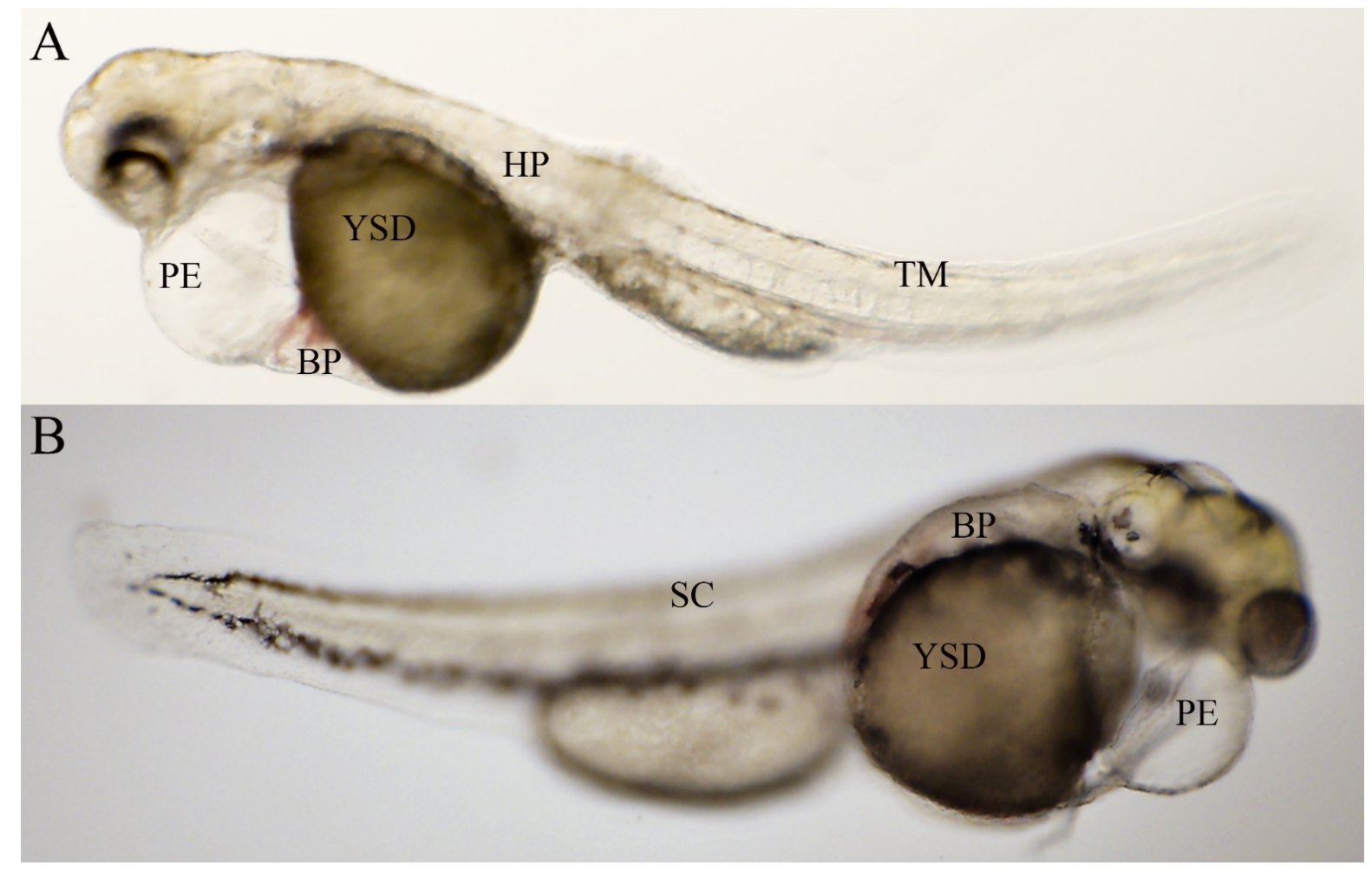

FIGURE 7: Day 3 representative embryos exposed to nTx ED and nTx DSG methacrylates: PE Pericardial edemata; BP Blood pooling YSD Yolk sac resorption delay HP Hypopigmented body including eye; SC Spinal curvature; TM Tail malformation.

\subsection{Effect of ethanol on biological performance of methacrylates}

The observed level of toxicity in the methacrylates can probably be explained by uncured monomers. This class of materials has high cytotoxicity due to Michael addition of the methacrylate group with amino or thiol groups of DNA or proteins in the human body. Similarly, hydrolysis of non-reacted groups forms unwanted methacrylic acid, decreasing $\mathrm{pH}$ locally, which may produce adverse biological effects on the surrounding tissue[41, 42]. By applying post-illumination, methacrylate conversion could be increased to improve biocompatibility.

Acrylic-based formulations are preferred in UV curing systems, because of the high reactivity of the acrylate double bond. The photoinitiators in the resins undergo fast homolytic cleavage upon intense illumination according to a Norrish-type I mechanism, to generate free radicals 
that will initiate the polymerization [37]. As polymerization progresses, the amount of aliphatic double bonds decreases but in practice, the process does not guarantee a $100 \%$ monomer conversion rate [43]. For this reason, different chemical and physical techniques exist that use temperature, initiator, reactive co-monomer, chemical removal, radiation, devolatilization and ion exchange exist to reduce residual monomer content in polymers, depending on their composition [44]. For this study, we observed 'detoxicant' effects of ethanol treatment. Immersion of dental acrylics in pure ethanol solution reduces residual compounds [45]. Likewise, ethanol-water solutions lower residual monomer contents in acrylic resins, depending on concentration and temperature[46]. Interestingly, poly methyl methacrylate dissolves in ethanol-aqueous solutions but it is insoluble in both water and ethanol at ambient temperature [47]. The ethanoic-aqueous effect may be due to induced swelling in polymeric chains, which allows insoluble substances to diffuse, partly due to increased water sorption[46]. Dissolution in polymers is preceded by heavy swelling and those bearing polar, hydrophilic groups may finally dissolve in polar solvents [48], hence caution is required in the use of ethanol to avoid its deleterious effects on structural integrity [49].

The varying conversion rates observed is possibly due to the chemical structure, functionality and concentration of the various constituents of the photopolymers [50]. ED composed of $>60 \%$ BisEMA recorded conversion rates of $22.33 \%$ and $27.10 \%$ in $\mathrm{nTx}$ and $\mathrm{Tx}$ conditions, respectively. DSG composed of $\geq 75 \%$ BisEMA recorded $20.88 \%$ and $31.12 \%$, in $\mathrm{nTx}$ and $\mathrm{Tx}$ conditions, respectively. The increased conversion rate in Tx methacrylates is consistent with the influence of ethanol on dimethacrylates [51]; however, dimethacrylates generally have lower conversion rates and the higher their composition, the lower the DC of the resulting polymer. The speedy curing rates observed in ED and EG liquid photopolymer resins (FIGURE 6) also correlate with their lower DC[7]. 


\subsection{Conclusions}

Despite the twofold increase in DC (\%) for nTx EG, it was unsafe in zebrafish bioassays, hence there is limited correlation between conversion rate and biological performance. The study affirms that biocompatibility was influenced primarily by physico-chemical characteristics of the materials, which subsequently influenced their residual monomer content before and after immersion in ethanol. Given the precautionary implications of the study, we propose a 3-tiered approach i.e. using approved materials, apposite manufacturing parameters and post-processing techniques that together guarantee optimal results for medical devices. With the current influx of $3 \mathrm{D}$ printers and materials on the market, it is imperative that buying decision is not limited to economic factors but rather, what will deliver long-term benefits. It is recommended that manufacturers set out rigorous treatment protocols for materials indicated medical devices and include the information in the literature that accompanies their products. Lastly, the zebrafish bioassay as a reliable toxicological screening tool could add to existing biological evaluation tests in dentistry, where a host of devices for prosthodontic treatments is constructed with methacrylates.

\section{Acknowledgements}

We thank EnvisionTec GmbH, Germany for kindly supplying E-Denture and E-Guard materials for our experiments. The study was partly supported by Australian Dental Research Foundation Grant (231-2017) and Griffith University School of Dentistry and Oral Health Grants. G.J.L was supported by the NHMRC (1044754, 1069284). The Australian Regenerative Medicine Institute is supported by funds from the State Government of Victoria and the Australian Federal Government.

\section{Conflict of interest statement}

No competing financial interests exist. 


\section{References}

[1] J.R. Fried, Polymer science and technology, Pearson Education, Inc., Upper Saddle River, 2014, pp. 1-24.

[2] A. Ravve, Principles of Polymer Chemistry, Springer, New York, 2012, pp. 1-15.

[3] Food and Drug Administration, Technical Considerations for Additive

Manufactured Devices:Draft Guidance for Industry and Food and Drug Administration

Staff, Food and Drug Administration, Rockville, MD:, 2016, pp. 1-28.

[4] G.N. Mir, W.H. Lawrence, J. Autian, Toxicological and pharmacological actions of methacrylate monomers I: Effects on isolated, perfused rabbit heart, Journal of Pharmaceutical Sciences 62(5) (1973) 778-782.

[5] G.N. Mir, W.H. Lawrence, J. Autian, Toxicological and pharmacological actions of methacrylate monomers II: Effects on isolated guinea pig ileum, Journal of Pharmaceutical Sciences 62(8) (1973) 1258-1261.

[6] G.N. Mir, W.H. Lawrence, J. Autian, Toxicological and pharmacological actions of methacrylate monomers III: Effects on respiratory and cardiovascular functions of anesthetized dogs, Journal of Pharmaceutical Sciences 63(3) (1974) 376-381.

[7] S.-H. Kim, D.C. Watts, Degree of conversion of bis-acrylic based provisional crown and fixed partial denture materials, The Journal of Korean Academy of Prosthodontics 46(6) (2008) 639-643.

[8] J. Autian, Structure-toxicity relationships of acrylic monomers, Environmental Health Perspectives 11 (1975) 141-152.

[9] K.L. Dearfield, C.S. Millis, K. Harrington-Brock, C.L. Doerr, M.M. Moore, Analysis of the genotoxicity of nine acrylate/methacrylate compounds in L5178Y mouse lymphoma cells, Mutagenesis 4(5) (1989) 381-393.

[10] K.H. Reinert, Aquatic toxicity of acrylates and methacrylates: Quantitative structure-activity relationships based on Kow and LC50, Regulatory Toxicology and Pharmacology 7(4) (1987) 384-389.

[11] C.L. Russom, R.A. Drummond, A.D. Huffman, Acute toxicity and behavioral effects of acrylates and methacrylates to juvenile fathead minnows, Bulletin of Environmental Contamination and Toxicology 41(4) (1988) 589-596. 
[12] M.M. Moore, A. Amtower, C.L. Doerr, K.H. Brock, K.L. Dearfield, Genotoxicity of acrylic acid, methyl acrylate, ethyl acrylate, methyl methacrylate, and ethyl methacrylate in L5178Y mouse lymphoma cells, Environmental and molecular mutagenesis 11(1) (1988) 49-63.

[13] Organisation for Economic Co-operation and Development, Test No. 236: Fish Embryo Acute Toxicity (FET) Test, OECD Publishing, Paris, 2013, pp. 1-22.

[14] T. Braunbeck, B. Kais, E. Lammer, J. Otte, K. Schneider, D. Stengel, R. Strecker, The fish embryo test (FET): origin, applications, and future, Environmental Science and Pollution Research 22(21) (2015) 16247-16261.

[15] F. Alifui-Segbaya, S. Varma, G.J. Lieschke, R. George, Biocompatibility of Photopolymers in 3D Printing, 3d Print Addit Manuf 4(4) (2017) 185-191.

[16] S. Richter, Medical Device Polymer Biocompatibility Screening Test Using Zebrafish Embryos, 2014. https://www.mdtmag.com/article/2014/03/medical-devicepolymer-biocompatibility-screening-test-using-zebrafish-embryos. (Accessed 22 November 2017).

[17] S.M. Oskui, G. Diamante, C. Liao, W. Shi, J. Gan, D. Schlenk, W.H. Grover, Assessing and Reducing the Toxicity of 3D-Printed Parts, Environmental Science \& Technology Letters 3(1) (2016) 1-6.

[18] N.P. Macdonald, F. Zhu, C.J. Hall, J. Reboud, P.S. Crosier, E.E. Patton, D. Wlodkowic, J.M. Cooper, Assessment of biocompatibility of 3D printed photopolymers using zebrafish embryo toxicity assays, Lab on a Chip 16(2) (2016) 291-297.

[19] F. Zhu, T. Friedrich, D. Nugegoda, J. Kaslin, D. Wlodkowic, Assessment of the biocompatibility of three-dimensional-printed polymers using multispecies toxicity tests, Biomicrofluidics 9(6) (2015).

[20] F. Zhu, N.P. Macdonald, J. Skommer, D. Wlodkowic, Biological implications of lab-on-a-chip devices fabricated using multi-jet modelling and stereolithography processes, SPIE Microtechnologies, SPIE, 2015, p. 9.

[21] F. Zhu, J. Skommer, N.P. Macdonald, T. Friedrich, J. Kaslin, D. Wlodkowic, Three-dimensional printed millifluidic devices for zebrafish embryo tests, Biomicrofluidics 9(4) (2015) 046502. 
[22] F. Zhu, J. Skommer, T. Friedrich, J. Kaslin, D. Wlodkowic, 3D printed polymers toxicity profiling: a caution for biodevice applications, SPIE Micro+Nano Materials, Devices, and Applications, SPIE, 2015, p. 7.

[23] K. Howe, M.D. Clark, C.F. Torroja, J. Torrance, C. Berthelot, M. Muffato, J.E. Collins, S. Humphray, K. McLaren, L. Matthews, S. McLaren, I. Sealy, M. Caccamo, C. Churcher, C. Scott, J.C. Barrett, R. Koch, G.-J. Rauch, S. White, W. Chow, B. Kilian, L.T. Quintais, J.A. Guerra-Assuncao, Y. Zhou, Y. Gu, J. Yen, J.-H. Vogel, T. Eyre, S. Redmond, R. Banerjee, J. Chi, B. Fu, E. Langley, S.F. Maguire, G.K. Laird, D. Lloyd, E. Kenyon, S. Donaldson, H. Sehra, J. Almeida-King, J. Loveland, S. Trevanion, M. Jones, M. Quail, D. Willey, A. Hunt, J. Burton, S. Sims, K. McLay, B. Plumb, J. Davis, C. Clee, K. Oliver, R. Clark, C. Riddle, D. Eliott, G. Threadgold, G. Harden, D. Ware, B. Mortimer, G. Kerry, P. Heath, B. Phillimore, A. Tracey, N. Corby, M. Dunn, C. Johnson, J. Wood, S. Clark, S. Pelan, G. Griffiths, M. Smith, R. Glithero, P. Howden, N. Barker, C. Stevens, J. Harley, K. Holt, G. Panagiotidis, J. Lovell, H. Beasley, C. Henderson, D. Gordon, K. Auger, D. Wright, J. Collins, C. Raisen, L. Dyer, K. Leung, L. Robertson, K. Ambridge, D. Leongamornlert, S. McGuire, R. Gilderthorp, C. Griffiths, D. Manthravadi, S. Nichol, G. Barker, S. Whitehead, M. Kay, J. Brown, C. Murnane, E. Gray, M. Humphries, N. Sycamore, D. Barker, D. Saunders, J. Wallis, A. Babbage, S. Hammond, M. Mashreghi-Mohammadi, L. Barr, S. Martin, P. Wray, A. Ellington, N. Matthews, M. Ellwood, R. Woodmansey, G. Clark, J. Cooper, A. Tromans, D. Grafham, C. Skuce, R. Pandian, R. Andrews, E. Harrison, A. Kimberley, J. Garnett, N. Fosker, R. Hall, P. Garner, D. Kelly, C. Bird, S. Palmer, I. Gehring, A. Berger, C.M. Dooley, Z. Ersan-Urun, C. Eser, H. Geiger, M. Geisler, L. Karotki, A. Kirn, J. Konantz, M. Konantz, M. Oberlander, S. Rudolph-Geiger, M. Teucke, K. Osoegawa, B. Zhu, A. Rapp, S. Widaa, C. Langford, F. Yang, N.P. Carter, J. Harrow, Z. Ning, J. Herrero, S.M.J. Searle, A. Enright, R. Geisler, R.H.A. Plasterk, C. Lee, M. Westerfield, P.J. de Jong, L.I. Zon, J.H. Postlethwait, C. Nusslein-Volhard, T.J.P. Hubbard, H.R. Crollius, J. Rogers, D.L. Stemple, The zebrafish reference genome sequence and its relationship to the human genome, Nature 496(7446) (2013) 498-503. [24] G.J. Lieschke, P.D. Currie, Animal models of human disease: zebrafish swim into view, Nat Rev Genet 8(5) (2007) 353-367. 
[25] EnvisionTec GmbH, Understanding the technology behind our advanced 3D printers., Advanced DLP for superior printing., 2016, pp. 1-7.

[26] Formlabs, Expanding 3D printing technologies to high-volume applications and beyond., Mary Ann Liebert, Inc. publishers, pp. 1-53.

[27] EnvisionTEC GmbH, E-Denture SDS ID: SENP2201701UK, 2017. https://envisiontec.com/wp-content/uploads/2018/02/SDS_EnvisionTec-E-Denture-EDent-400-E-Guide-Tint_SENP2201701UK-1.pdf. (Accessed 18 May 2018).

[28] EnvisionTEC GmbH, E-Guard SDS ID: M-NOC-2015-01-UK, 2015. https://envisiontec.com/wp-content/uploads/2017/08/SDS-E-Guard.pdf. (Accessed 13 May 2018).

[29] Formlabs, Safety Data Sheet: Dental SG, 2018. https://support.formlabs.com/hc/enus/articles/115000015710-Using-Dental-SG-Resin. (Accessed 14 June 2018).

[30] Formlabs, Dental SG: Material properties, 2017.

https://formlabs.com/media/upload/DentalSG-DataSheet.pdf. (Accessed 24 July 2017).

[31] International Organization for Standardization, ISO 10993-12: Biological evaluation of medical devices - Part 12: Sample preparation and reference materials, International Organization for Standardization, Geneva, 2012.

[32] R. Nagel, DarT: The embryo test with the zebrafish Danio rerio - a general model in ecotoxicology and toxicology, Altex-Altern Tierexp 19 (2002) 38-48.

[33] International Organization for Standardization, ISO 10993-5, Biological evaluation of medical devices - Part 5: Tests for in vitro cytotoxicity, International Organization for Standardization, Geneva, 2009.

[34] Food and Drug Administration, NextDent ${ }^{\mathrm{TM}}$ Denture / E-Denture 3D-printing material, 2017. https://www.accessdata.fda.gov/cdrh_docs/pdf16/k162572.pdf.

(Accessed 18 January 2018).

[35] Formlab, Dental LT Clear Technical Data Sheet and Safety Data Sheet, 2017. https://support.formlabs.com/hc/en-us/articles/115001530544-Printing-Splints-withDental-LT-Clear-Resin. (Accessed 7 June 2018).

[36] I. Sideridou, V. Tserki, G. Papanastasiou, Effect of chemical structure on degree of conversion in light-cured dimethacrylate-based dental resins, Biomaterials 23(8) (2002) 1819-1829. 
[37] C. Decker, Kinetic Study and New Applications of UV Radiation Curing, Macromolecular Rapid Communications 23(18) (2002) 1067-1093.

[38] W.H. Lawrence, J. Autian, Possible Toxic Effects from Inhalation of Dental Ingredients by Alteration of Drug Biologic Half-Life, Journal of Dental Research 51(3) (1972) 878-878.

[39] M. Westerfield, The Zebrafish Book. A Guide for the Laboratory Use of Zebrafish (Danio rerio), University of Oregon Press, Eugene, 2000.

[40] Organisation for Economic Co-operation and Development, Test No. 210: Fish, Early-life Stage Toxicity Test, OECD Publishing, Paris, 2013, pp. 1-24.

[41] B. Husár, M. Hatzenbichler, V. Mironov, R. Liska, J. Stampfl, A. Ovsianikov, 6 Photopolymerization-based additive manufacturing for the development of 3D porous scaffolds, Biomaterials for Bone Regeneration, Woodhead Publishing2014, pp. 149201.

[42] L.S. Andrews, J.J. Clary, Review of the toxicity of multifunctional acrylates, Journal of toxicology and environmental health 19(2) (1986) 149-64.

[43] S.J. Duray, J.L. Gilbert, E.P. Lautenschlager, Comparison of chemical analysis of residual monomer in a chemical-cured dental acrylic material to an FTIR method, Dental Materials 13(4) (1997) 240-245.

[44] P.H.H. Araújo, C. Sayer, R. Giudici, J.G.R. Poço, Techniques for reducing residual monomer content in polymers: A review, Polymer Engineering \& Science 42(7) (2002) $1442-1468$.

[45] A.F. Boeckler, D. Morton, S. Poser, K.-E. Dette, Release of dibenzoyl peroxide from polymethyl methacrylate denture base resins: An in vitro evaluation, Dental Materials 24(12) (2008) 1602-1607.

[46] C.B. Neves, L.P. Lopes, H.F. Ferrao, J.P. Miranda, M.F. Castro, A.F. Bettencourt, Ethanol postpolymerization treatment for improving the biocompatibility of acrylic reline resins, BioMed research international 2013 (2013) 485246.

[47] R. Hoogenboom, C.R. Becer, C. Guerrero-Sanchez, S. Hoeppener, U.S. Schubert, Solubility and Thermoresponsiveness of PMMA in Alcohol-Water Solvent Mixtures, Australian Journal of Chemistry 63(8) (2010) 1173-1178.

[48] P. Ghosh, Fundamentals of Polymer Science: Solution Properties, 2006. 
[49] P.-P. Wang, S. Lee, J.P. Harmon, Ethanol-induced crack healing in poly(methyl methacrylate), Journal of Polymer Science Part B: Polymer Physics 32(7) (1994) 12171227.

[50] C. Decker, Photoinitiated crosslinking polymerisation, Progress in Polymer Science 21(4) (1996) 593-650.

[51] J. Malacarne-Zanon, D.H. Pashley, K.A. Agee, S. Foulger, M.C. Alves, L. Breschi, M. Cadenaro, F.P. Garcia, M.R. Carrilho, Effects of ethanol addition on the water sorption/solubility and percent conversion of comonomers in model dental adhesives, Dental materials : official publication of the Academy of Dental Materials 25(10) (2009) 10.1016/j.dental.2009.03.015. 
This page intentionally left blank 